\title{
Análisis De Tipologías De Construcciones Alternativas Aplicadas A La Construcción De Un Centro Cultural Riobamba-Ecuador
}

Carlos Renato Chávez Velásquez

Docente investigador - Ingeniero en Ecoturismo

Escuela Superior Politécnica de Chimborazo

Tecnólogo en Construcciones Andinas

Francisco Cepeda Yautibug

Docente investigador

Instituto Superior "Jatun Yachay Wasi" Colta - Ecuador

Luis Enrique Ávila Granda

Docente investigador

Instituto Superior "Jatun Yachay Wasi" Colta - Ecuador

Wilian Ernesto Balseca del Valle

Docente investigador

Instituto Superior "Jatun Yachay Wasi" Colta - Ecuador

Juan Enrique Ureña Moreno

Docente investigador - Ingeniero civil

Escuela Superior Politécnica de Chimborazo

Sofía Andrea Basantes Vinueza

Ingeniera en Ecoturismo

Escuela Superior Politécnica de Chimborazo

doi: 10.19044/esj.2017.v13n18p73 URL:http://dx.doi.org/10.19044/esj.2017.v13n18p73

Abstract

The traditional construction on land in the Ecuadorian highlands has been displaced by new construction methodologies and materials. The present study shows in detail the most used vernacular materials, describing their potential and comparing them accurately to the contemporary traditional solid brick. The modeling's of each of the materials were executed/built/created in a small area of the Cultural Center of the Polytechnic School of Chimborazo. This was possible to do by using specialized software that details the thermal behavior and by knowing in advance the variations of temperature throughout the day and night in the Ecuadorian highlands. The Cultural Center is a center that opens a space for 
the exchange of intercultural knowledge and aims to teach the importance of sustainability by using and optimizing resources. This research aims to present the alternative of building thermal comfort buildings with sustainable materials within the university campus. These building will represent a space for teaching and learning among students, teachers and visitors who wish to learn about Cultural Heritage, Natural Resources, Sustainability, etc. Finally, this research contributes to the higher education system in the university as it goes alone with the teaching objectives established in each School, especially in the school of Ecotourism.

Keywords: Traditional construction, Sustainability, Thermal comfort, Cultural center, Tourism and culture.

\section{Resumen}

La construcción en tierra usada en el transcurso de décadas en la serranía ecuatoriana ha sido desplazada por nuevas metodologías constructivas y materiales. El presente estudio detalla los materiales vernáculos más usados describiendo sus potencialidades para después compararlos precisa y detalladamente con el material contemporáneo insigne de la nueva envolvente como es el ladrillo cocido macizo artesanal. Con el uso de un software especializado que detalla el comportamiento térmico, conociendo de antemano de las variaciones de temperatura a lo largo del día y de la noche en la serranía ecuatoriana, se ejecutan modelaciones de cada uno de los materiales tomando como escenario una pequeña zona del complejo en el marco de la implementación de un Centro Cultural en la Escuela Superior Politécnica de Chimborazo que responde a la idea general de construir un espacio de intercambio de saberes, con el propósito de enseñar también sobre sostenibilidad, esto último ejemplificándolo en la utilización y optimización de recursos. Se pretende con esta investigación poner a consideración la alternativa de construir edificios térmicamente confortables con materiales heredados en las Universidades, que a su vez representen un espacio de enseñanza y convivencia entre los estudiantes, los maestros y los visitantes que deseen conocer sobre temas de Patrimonio Cultural, Recursos Naturales, Sostenibilidad entre otros. Finalmente es necesario el aporte de la investigación en el área de Educación Superior, más aun cuando se responde a los objetivos mismos de estudio y enseñanza de cada carrera como por ejemplo la carrera de Ecoturismo.

Palabras Clave: Tipologías de construcciones - Sostenibilidad - confort térmico - Centro Cultural - Turismo y cultura 


\section{Introduccion}

El buen manejo de los desechos sólidos y la obtención de productos orgánicos, son dos de los temas que se analizan para optimizar los espacios y mejorar la sostenibilidad de los territorios que habitamos, además otro aspecto que se debe analizar es, la vivienda, con el propósito de favorecer al ámbito físico - ambiental que el planeta necesita para lograr un equilibrio, por ello los lugares que habitamos ya sean casas, escuelas, universidades, edificios gubernamentales y otras áreas; son también espacios que se pueden adaptar, cambiar y convertirse en construcciones sostenibles para el mejor uso de los recursos a la vez que se armonice con el medio que nos rodea (Acosta, 2009, pág. 15).

Partiendo del concepto básico establecido por la UNESCO (2005), el cual indica que "desarrollo sostenible es aquel que satisface las necesidades de las generaciones presentes sin comprometer la capacidad de las generaciones futuras para satisfacer sus propias necesidades", se considera que es un concepto sutil, ya que deja varios ámbitos de análisis sin abordar, entre otros casos para futuras y presentes investigaciones este concepto se adapta, por lo que la palabra sostenibilidad se debe entender que abarca los ámbitos social, económico, ambiental y cultural.

Pragmáticamente se debe entender que al mejorar la utilización de los recursos que tenemos a disposición, así como optimizar las técnicas que ya demostraron ser eficaces en tiempos predecesores mediante la tecnología y la investigación, permiten potencializar las construcciones con materiales alternativos como la tierra, adobe, tapial entre otros (Bedoya, 2011).

Partiendo desde los tiempos prehistóricos, poblaciones de indios norteamericanos y de tribus en África, ciudades desarrolladas europeas y pueblos prósperos en América, poseen la característica conjunta de haber utilizado la tierra como material y recurso práctico para la construcción de sus viviendas. Esto se refleja al encontrar en una extensa bibliografía sobre este tópico, tipologías de sistemas constructivos basados en la utilización de la tierra como materia prima; Egipto, Marruecos, Francia, España, Colombia, México, Perú, Ecuador y otros países, contemplan o han contemplado este tipo de construcción milenaria (Del Río M., Sainz A., 2012).

De acuerdo a Bedoya (2011), la tierra como materia prima representa una opción práctica por su versatilidad que le permite ser empleada con pocas limitaciones espaciales; económica, ya que su extracción puede hacerse en el mismo sitio de la construcción, evitando transportes de materias primas; y ambiental, al ser empleado como material de construcción, elimina el transporte de residuos, al mismo tiempo que evita que esa misma cantidad sea extraída de canteras como recurso no renovable.

Para la presente investigación se analizaron las técnicas de construcciones alternativas que se pueden emplear con el objetivo de aportar 
al desarrollo de la sostenibilidad, promoviéndolo desde el área formativa. Como estudio de caso se tiene la Construcción de un Centro Cultural en la ESPOCH, aplicando varios métodos que se han perdido en el trascurso de los años pero que siguen siendo efectivos en la región sierra centro del Ecuador.

En un primer apartado para el desarrollo de la presente investigación se especifica la metodología que se utilizó y el diagnóstico que se realizó para la implementación del Centro Cultural, el mismo que cumple con el objetivo de ser un espacio para el estudio y la difusión del Patrimonio Cultural de las provincias de la región sierra centro del Ecuador. A la vez se pretende con este estudio promover técnicas alternativas en la construcción, que permitan poner en práctica la misión y visión de la Facultad de Recursos Naturales de la ESPOCH, en consecuencia se analizan los tipos de materiales y el proceso de elaboración así como el análisis de las fortalezas constructivas y la conductividad térmica de los mismos.

\section{Propuesta para la implementación del Centro Cultural en la Escuela Superior Politécnica de Chimborazo}

La investigación realizada sobre el "Diseño de un Centro Cultural para la Escuela de Ingeniería en Ecoturismo, Facultad de Recursos Naturales de la Escuela Superior Politécnica de Chimborazo", contempló el estudio de la viabilidad económica-financiera, estudio administrativo-legal y el estudio más extenso de la viabilidad comercial. Estos resultados se exponen en otro apartado por estar indirectamente relacionados con el tema central de este documento que es "Tipologías de Construcciones Alternativas", sin embargo para mayor información referirse a la Tesis para la obtención del título de Ingeniera en Ecoturismo de la autora Jenny Álvarez año 2015.

Para iniciar con la investigación, se elaboró un diagnóstico situacional académico del área de gestión turística del patrimonio cultural de la Escuela de Ingeniería en Ecoturismo, para lo cual se consideró dos funciones principales que deben tener las instituciones de educación superior, como son: docencia y la investigación.

Para elaborar el diagnóstico académico en el área de docencia se realizaron entrevistas a los miembros de la Comisión de Carrera de la Escuela de Ingeniería en Ecoturismo y encuestas a los docentes del área de gestión del patrimonio cultural, para identificar y analizar los requerimientos y necesidades pedagógicas y didácticas establecidas en los programas analíticos (sílabos), de las asignaturas afines para impartir las diferentes cátedras, esto con el objetivo de mejorar el proceso de aprendizaje en la escuela. De igual manera se realizaron encuestas a los docentes que están ejecutando proyectos de investigación en el área de patrimonio cultural, con la finalidad de identificar las necesidades y requerimientos científicos y 
tecnológicos para el registro, inventariación y salvaguardia del patrimonio cultural.

\section{Planificación y diseño técnico del Centro Cultural}

El Centro Cultural será creado para brindar a los estudiantes escolares, secundarios y universitarios del país, una alternativa para ampliar los conocimientos sobre patrimonio cultural, además de transformarse en una fuente de conocimiento para los turistas locales, nacionales y extranjeros, obteniendo una información completa del patrimonio cultural de la región Sierra centro.

\section{Localización del proyecto}

Macrolocalización: El proyecto se ubicará en el cantón Riobamba

Microlocalización: El proyecto se ubicará en la Escuela Superior Politécnica de Chimborazo en los predios de la Facultad de Recursos Naturales.

Tamaño del proyecto.- Para el tamaño del proyecto se tomó en cuenta el área de construcción más las áreas verdes y recreación que es un total de $18726.86 \mathrm{~m}^{2}$ con una capacidad física de 1000 personas a la vez.

Tabla 1. Distribución de espacios para el centro cultural

\begin{tabular}{|c|c|}
\hline Áreas & Detalle \\
\hline \multirow{10}{*}{ Área 1} & Ingreso principal \\
\hline & Guardianía \\
\hline & Plaza de acceso \\
\hline & Parqueaderos \\
\hline & Administración \\
\hline & Bodega General \\
\hline & Biblioteca y Videoteca \\
\hline & Bloque de baterías sanitarias \\
\hline & Salas de Interpretación \\
\hline & Laboratorios \\
\hline \multirow{4}{*}{ Área 2} & Talleres de Uso Múltiple \\
\hline & Cafetería \\
\hline & Zona de Exposición Exterior y recreación \\
\hline & Zona de Lectura y Descanso \\
\hline \multirow{2}{*}{ Área 3} & Aulas Talleres \\
\hline & Baterías Sanitarias \\
\hline $\begin{array}{c}\text { Área de } \\
\text { Sustentabilidad } \\
\end{array}$ & $\begin{array}{l}\text { Disposición de energías alternativas: } \\
\text { Eólica y solar }\end{array}$ \\
\hline
\end{tabular}

Nota: Tesis Jenny Álvarez, 2015 


\section{Distribución de la construcción}

Tabla 2. Descripción de cada área

\begin{tabular}{|c|c|}
\hline Área 1 & Descripción del ambiente \\
\hline Ingreso principal & $\begin{array}{l}\text { Estará provista para el ingreso al centro con rampas de acceso en caso } \\
\text { de que se tenga la presencia de turistas con capacidades especiales }\end{array}$ \\
\hline Guardianía & Estará provista de una caseta para guardia con registros de visita. \\
\hline Plaza de acceso & Facilitará el acceso a los visitantes \\
\hline Parqueaderos & $\begin{array}{l}\text { Contará con un área de } 500 \mathrm{~m}^{2} \text { con una capacidad para } 20 \text { parqueos } \\
\text { incluido para personas con capacidades diferentes. }\end{array}$ \\
\hline Administración & $\begin{array}{l}\text { Lugar donde se encontrará el personal administrativo, lugar donde se } \\
\text { realizará reuniones con todo el personal que labore dentro del Centro } \\
\text { Cultural, para planificación y toma de decisiones. Este espacio } \\
\text { dispondrá de oficinas con su respectivo mobiliario. Contará con un } \\
\text { área de } 52,5 \mathrm{~m}^{2} \text { y estará provista de baterías sanitarias. }\end{array}$ \\
\hline Bodega general & $\begin{array}{l}\text { Contará con un área de } 25 \mathrm{~m}^{2} \text {, estará provista de perchas para el } \\
\text { correcto almacenamiento de objetos adquiridos por el centro para su } \\
\text { óptimo funcionamiento. }\end{array}$ \\
\hline $\begin{array}{l}\text { Biblioteca } \\
\text { videoteca }\end{array}$ & $\begin{array}{l}\text { Contará con un área de } 325 \mathrm{~m}^{2} \text { estará provista por espacios de lectura } \\
\text { e investigación, espacios de proyección de videos de índole cultural, y } \\
\text { áreas de audio con relatos del patrimonio cultural local, regional y } \\
\text { nacional. }\end{array}$ \\
\hline $\begin{array}{l}\text { Bloque } \\
\text { baterías } \\
\text { sanitarias }\end{array}$ & $\begin{array}{l}\text { Contará con un área de } 27 \mathrm{~m}^{2} \text {, estará provista de los insumos } \\
\text { necesarios para el aseo personal de los visitantes. }\end{array}$ \\
\hline $\begin{array}{l}\text { Salas de } \\
\text { Interpretación }\end{array}$ & $\begin{array}{l}\text { Contará con un área de } 160 \mathrm{~m}^{2} \text {, estará provista por una galería } \\
\text { fotográfica de los principales patrimonios culturales que identifique a } \\
\text { las provincias de Chimborazo, Tungurahua y Bolívar. Además de los } \\
\text { medios interpretativos. }\end{array}$ \\
\hline Laboratorio & $\begin{array}{l}\text { Contará con un área de } 37,40 \mathrm{~m}^{2} \text {, estará provisto de implementos para } \\
\text { la investigación de suelos, herramientas para levantamientos } \\
\text { arqueológicos y GPS. }\end{array}$ \\
\hline Área 2 & a del ambiente \\
\hline $\begin{array}{l}\text { Talleres de Uso } \\
\text { Múltiple }\end{array}$ & $\begin{array}{l}\text { Contará con un área de } 686 \mathrm{~m} 2 \text {, estará provisto de elementos que } \\
\text { faciliten las diferentes actividades a realizarse. }\end{array}$ \\
\hline Cafetería & $\begin{array}{l}\text { Contará con un área de } 276 \mathrm{~m} 2 \text {, estará provisto por el equipamiento } \\
\text { necesario para la correcta operación de los servicios. }\end{array}$ \\
\hline $\begin{array}{l}\text { Área } \\
\text { Exposición } \\
\text { Exterior } \\
\text { recreación }\end{array}$ & $\begin{array}{l}\text { Contará con un área de } 1896 \mathrm{~m} 2 \text {, estará provisto por una decoración } \\
\text { natural y contemporánea que contraste con el entorno. }\end{array}$ \\
\hline $\begin{array}{l}\text { Área de Lectura y } \\
\text { Descanso }\end{array}$ & $\begin{array}{l}\text { Contará con un área de } 800 \mathrm{~m} 2 \text {, estará provisto por una decoración } \\
\text { natural y contemporánea que contraste con el entorno. }\end{array}$ \\
\hline Área 3 & Descripción del ambiente \\
\hline Aulas Talleres & $\begin{array}{l}\text { Contará con un área de } 92 \mathrm{~m} 2 \text {, estará equipado como } 8 \text { aulas } \\
\text { tradicionales en la que se imparten clases cuya capacidad es de } 25 \\
\text { personas cada una. }\end{array}$ \\
\hline Baterías Sanitarias & $\begin{array}{l}\text { Contará con un área de } 40 \mathrm{~m} 2 \text {, estará equipado acorde a lo expuesto } \\
\text { por el MINTUR y MSP }\end{array}$ \\
\hline Zona & Contará con un área de $1708 \mathrm{~m} 2$, estará equipado por paneles solares \\
\hline
\end{tabular}


Sustentabilidad

y turbinas para captar la energía eólica. Generará energías alternativas para cumplir con los objetivos del Plan Nacional del Buen Vivir y Desarrollo Sostenible del Centro Cultural.

Nota: Tesis Jenny Álvarez, 2015

\section{Tipología de la construcción}

El Centro Cultural tendrá una construcción que se enmarca en un ámbito moderno, con elementos que ayudan a que la construcción se pueda mantener a sí misma como es la aplicación de energías alternativas solares y eólicas, y de la misma manera la recolección de aguas lluvias para su reutilización, entre varios aspectos que se detallan a continuación como es el análisis de los materiales con los cuales se edificaría el Centro.

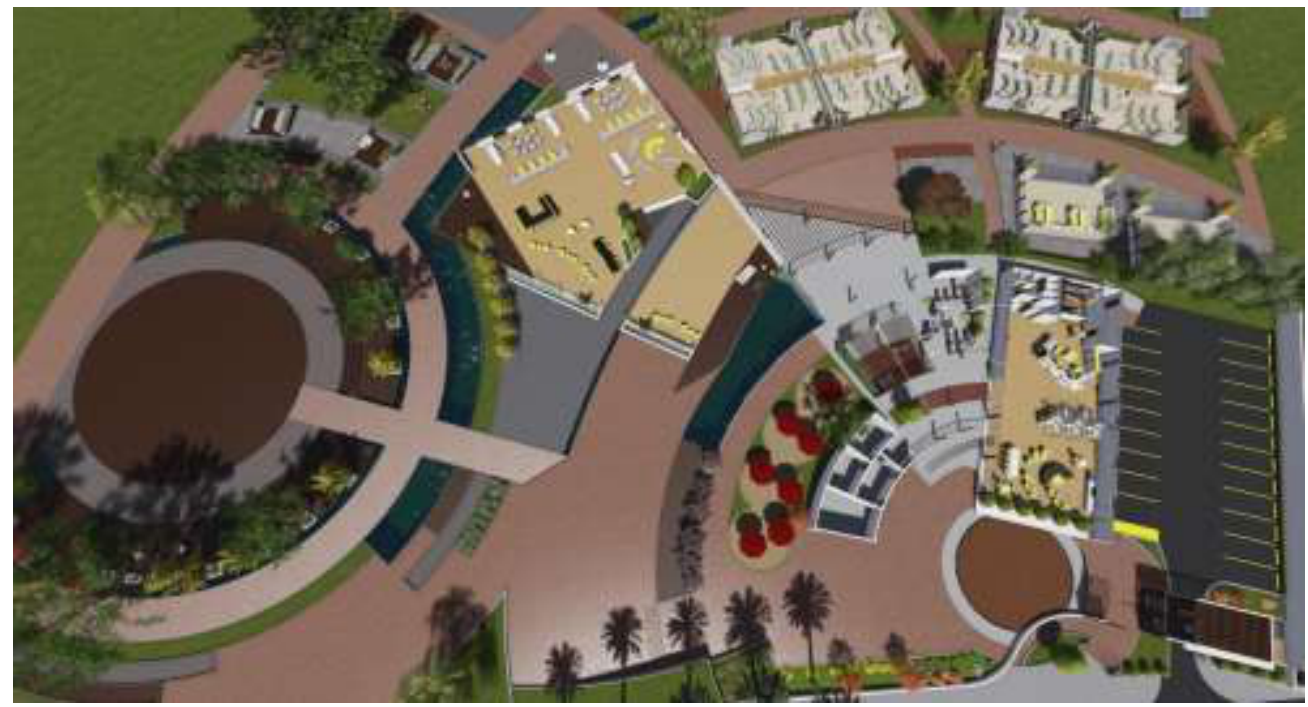

Imagen 1. Diseño en 3D de las instalaciones del Centro Cltural

Nota: Estudio técnico tomado de Tesis Jenny Alvarez, 2015

\section{Métodos de construcciones alternativas analizadas para la construcción} del centro cultural

Análisis y conceptos básicos sobre técnicas de construcciones con tierra

Para poder analizar qué tipo de técnica será la adecuada para la construcción del Centro Cultural se definirán y se explicarán varios conceptos de diferentes autores sobre el proceso de preparación para cada tipo de tecnologías de construcción con tierra, a la vez de una breve introducción sobre los orígenes de la misma. Luego se analizará sobre los aspectos básicos que se requieren y el lugar de construcción del Centro Cultural, comparando los beneficios y las desventajas de cada una de las técnicas.

Entre los elementos más destacados para la utilización en la región sierra centro del Ecuador, encontramos varios materiales y técnicas de construcción que son propios de la zona, recalcando que se utiliza los 
elementos que la misma área de construcción proporciona. Es así que se puede construir con la tierra que a su vez debe pasar por un proceso previo de preparación, dependiendo de la consistencia y la técnica que se desee emplear, se utiliza también madera de eucalipto, la planta de paja proveniente del páramo y otros elementos como la cal, desechos orgánicos de animales y ciertas plantas que dependiendo de su empleo le dan cierto acabado a la construcción.

\section{Adobe}

Es también conocido como ladrillo de tierra. La palabra adobe proviene de la palabra árabe atob, que significa ladrillo secado al sol. Su uso data de muchos miles de años atrás, la utilización de un molde para conformar las piezas de adobe fue probablemente llevado a España desde África y traído al territorio que ahora ocupa Estados Unidos por los conquistadores españoles en el siglo XVI (Bedoya, 2011, pág. 55).

$\mathrm{Su}$ diferencia con el ladrillo que actualmente en muy usado en la construcción, radica en que el adobe no se somete a proceso de cocción, a diferencia del ladrillo actual, que a través de temperaturas de entre $800^{\circ} \mathrm{C}$ y $1000{ }^{\circ} \mathrm{C}$, se convierte en un material cerámico más duro y de buen comportamiento en cuanto a la erosión. Las piezas de tierra cruda, como también se le conoce a esta técnica, son secadas en condiciones atmosféricas normales, a diferencia del ladrillo cerámico, el adobe puede ser confeccionado sin necesidad de prensas para la compresión o la extrusión, requiriendo sólo de moldes para verter en su interior la tierra.

Lo más positivo de la fabricación de adobes para la construcción de viviendas, bodegas o casas especiales de recreación, entre otras, es que la mayoría de terrenos son aptos para la elaboración de los prefabricados. Se recomienda, sin embargo, que la composición del suelo sea de un $55 \%$ a un $75 \%$ arena; de un $10 \%$ a un $28 \%$ de limo; y, de un $15 \%$ a un $18 \%$, de arcilla. Con estas composiciones se garantiza un material que presentará condiciones favorables para la manejabilidad, el curado y su desempeño posterior en obra. (Ibidem, 2011).

\section{Fabricación del adobe}

De acuerdo con el autor Oshiro (1995), se deben considerar los siguientes procedimientos para obtener un adobe firme y apto para la construcción de una edificación:

- La tierra con la que se elabore el adobe, debe ser limpia, sin piedras ni desperdicios.

- $\quad$ Debe contener un balance apropiado de arena y arcilla

- Los suelos arcillosos ocasionan demasiado encogimiento y rajaduras 
- Los suelos arenosos no tienen suficiente ligazón entre partículas, los adobes se desmoronan.

- Los suelos con excesivo contenido de material orgánico no son aptos para la construcción, por su baja resistencia y poca duración ante la humedad.

- Los suelos con sales solubles, atrae la humedad. Se debe rechazar esta clase de suelo.

Se deberá elegir el lugar donde se habrá de moldear los adobes, para esto se requiere de la disponibilidad de agua, además para determinar si el suelo es apropiado, deberá fabricarse adobes de prueba, de diferentes canteras, observar su comportamiento y elegir la tierra más apta.

Para la preparación del barro, una vez seleccionada la tierra deberá extraerse las piedras y elementos extraños. La tierra deberá ser humedecida totalmente y permanecer en reposo 1 o 2 días, antes de moldear.

El tendal es el proceso en el cual se prepara la zona para el secado del adobe. Esta zona deberá estar limpia, nivelada y los suficientemente extensa para albergar la producción de varios días. Para evitar la adherencia del adobe y el suelo, se coloca una capa de arena fina, papel u otro material que pueda ser fácilmente separado del adobe.

El proceso del moldeo permite la preparación de moldes sin fondo o con fondo. El uso de molde sin fondo permite un mayor avance, pero se logra una mejor compactación pero a su vez mayor fatiga por parte del trabajador.

El molde se complementa con una regla empleada para "cortar" los excesos de barro. Después de cada uso, el molde deberá limpiarse y rociarse con arena para evitar que el barro se pegue. El tiempo de secado depende del clima pudiendo tardar de 2 a 4 semanas. Cuando la consistencia de los adobes lo permita ( 3 a 5 días) deben colocarse de lado para asegurar un secado más rápido, posteriormente podrá apilarse, de manera que circule aire entre los adobes, hasta completar su secado.

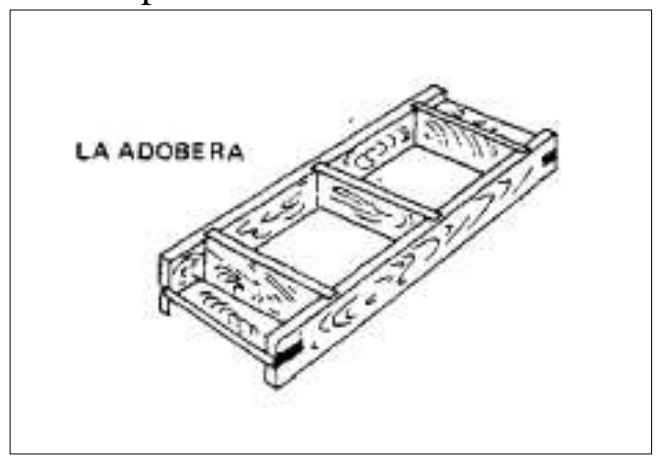

Fig. 1. Forma de la adobera

Nota: Construcción de vivienda económica en adobe estabilizado, 1990. 


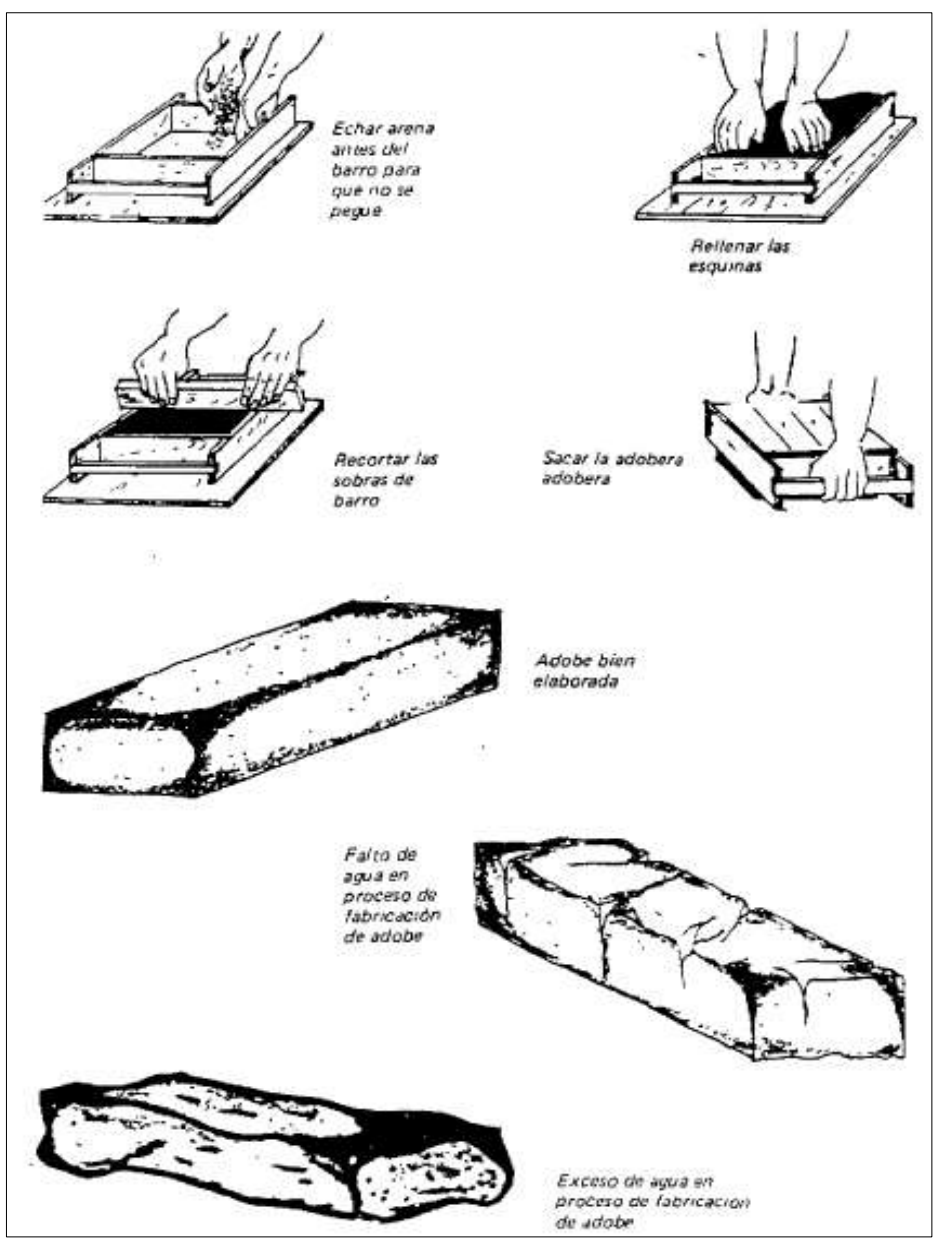

Fig. 2. Proceso de moldeado y ejemplo de adobes terminados

Nota: Construcción de vivienda económica en adobe estabilizado, 1990

\section{La tapia}

Se le conoce en varios países de América Latina con el nombre de tapia pisada. Este sistema constructivo consiste en disponer formaletas verticales y llenar su interior con tierra, compactándola manualmente por capas hasta formar muros macizos que tienen espesores entre $0,80 \mathrm{~m}$ y 0,50 m. La disponibilidad de tierras aptas para construir con la técnica de la tapia es alta, por no decir que total, pues casi todos los suelos poseen características favorables para derivar en un material de construcción, exceptuando aquellos que contienen ferro níquel o que obedecen estrictamente a minas, cuya composición superficial, no permite que el suelo tenga cohesión. (Bedoya, 2011, pág 59).

El tapial consiste en apisonar la tierra por tongadas (capas) entre dos planchas de madera (formaleta). Cuando se ha terminado una sección de 
muro se desplaza el encofrado hacia arriba o hacia un lado y se continúa la operación hasta su realización total. El encofrado puede hacerse manualmente con la ayuda de un pisón o utilizando una prensa mecánica. Las dimensiones más generalizadas del encofrado son de $300 \mathrm{~cm}$ de longitud, $90 \mathrm{~cm}$ de altura y $50 \mathrm{~cm}$ de ancho (Torroja, 1984, pág. 51)

\section{Bahareque}

Este es un sistema constructivo tendinoso, dado que funciona basado en una estructura de guadua recubierta con tierra, la cual puede estar mezclada con cagajón. Este tipo de construcción tiene gran uso a nivel rural. A diferencia de la tapia, el bahareque no emplea espesores tan considerables en los muros, ya que al configurar inicialmente una estructura tipo esqueleto con la guadua, hace que no se requiera de tanta inercia para soportar las solicitaciones externas de viento y sismos. Se figura el bahareque como un concreto reforzado natural, o tal vez, y quizás por historia, sería más justo decir que se figura el concreto reforzado como un bahareque artificial.

$\mathrm{Al}$ igual que la tapia, el bahareque ha experimentado una especie de discriminación como sistema constructivo por parte de la población actual, tanto a nivel rural como urbano. Se le considera un sistema anticuado, subdesarrollado y de mal comportamiento ante sismos; sin embargo, estos tres calificativos son incorrectos y, más que eso, injustos para un sistema de construcción que ofrece ventajas económicas y ambientales con respecto a los que fungen como modernos y desarrollados (Bedoya, 2011: 71).

Algunas de las ventajas de construir en bahareque son:

- $\quad$ La disposición abundante del material tierra y del cagajón de las bestias y demás animales.

- $\quad$ El ahorro en energía y transporte de materiales, sobre todo en áreas rurales alejadas de los centros urbanos.

- $\quad$ La fácil obtención de madera y guadua para erigir la estructura esqueletal de la vivienda o edificación.

- $\quad$ La inercia térmica que logra ambientes interiores confortables.

Las fortalezas constructivas para el proyecto de los materiales vernáculos se resumen en la siguiente tabla: 
Tabla 3. Comparación de fortalezas constructivas en el uso de materiales heredados

\begin{tabular}{|c|c|c|c|}
\hline MATERIAL & $\begin{array}{c}\text { Ladrillo de ADOBE } \\
\text { Espesor: } 40 \text { y } 20 \mathrm{~cm}\end{array}$ & TAPIAL & BAHAREQUE \\
\hline $\begin{array}{c}\text { Uso de Piezas } \\
\text { Monolíticas }\end{array}$ & $\sqrt{ }$ & $\times$ & $\times$ \\
\hline Mayor Inercia térmica & $\sqrt{ }$ & $\sqrt{ }$ & $\sqrt{ }$ \\
\hline Rapidez constructiva & $\sqrt{ }$ & $\sqrt{ }$ & $\sqrt{ }$ \\
\hline $\begin{array}{c}\text { Mano de obra } \\
\text { especializada }\end{array}$ & $\times$ & $\sqrt{ }$ & $\times$ \\
\hline $\begin{array}{c}\text { Posibilidad de un } \\
\text { Acabado estético }\end{array}$ & $\sqrt{ }$ & $\times$ & $\sqrt{ }$ \\
\hline Divisiones interiores & $\sqrt{ }$ & $\times$ & $\sqrt{ }$ \\
\hline $\begin{array}{c}\text { Comportamiento } \\
\text { sísmico }\end{array}$ & $\sqrt{ } *(1)$ & $\sqrt{ }$ \\
\hline Menor costo & $\sqrt{ }$ & & $\times$ \\
\hline
\end{tabular}

Por las características arquitectónicas del Centro de interpretación a más de características específicas de clima variable en el Ecuador continental se concluye que el Ladrillo de Adobe Monolítico es la mejor opción en materiales heredados como material de la envolvente de paredes para la consecución del proyecto.

El uso de mano de obra no especializada, espacios interiores amplios con el uso de bloques monolíticos, la gran inercia térmica y el bajo costo son características indiscutibles para su elección de entre los materiales mencionados.

La gran inercia térmica puede ser obtenida en valores calculados del muro de adobe enfoscado con yeso.

Determinando el coeficiente de transmitancia térmica " $K$ ":

$\mathrm{K}=1 / \mathrm{R}$

$\mathrm{R}=(1 / \alpha 1+\mathrm{e} 1 / \lambda 1+\mathrm{e} 2 / \lambda 2+\mathrm{e} 3 / \lambda 3+1 / \alpha \mathrm{e})$

Dónde:

e $2 / \lambda 2=e 3 / \lambda 3$ por método constructivo

$\alpha 1=$ Resistencia Superficial Interna

$\alpha e=$ Capa de aire superficial externa 
Tabla 4. Valores de conductividad térmica

\begin{tabular}{|c|c|c|c|}
\hline $\begin{array}{c}\text { Capa de elemento } \\
\text { constructivo (Paredes) }\end{array}$ & $\begin{array}{c}\text { Espesor } \\
\mathrm{e}(\mathrm{m})\end{array}$ & $\begin{array}{c}\text { Conductividad } \\
\text { térmica } \lambda(\mathrm{W} / \mathrm{mK})\end{array}$ & $\begin{array}{c}\text { Resistencia al paso } \\
\text { del calor } \\
\mathrm{R}\left(\mathrm{W} / \mathrm{m}^{2} \mathrm{~K}\right)\end{array}$ \\
\hline $\begin{array}{c}\text { Resistencia superficial } \\
\text { interna }\end{array}$ & ------------- & ------------- & 0.17 \\
\hline ENLUCIDO DE YESO & 0.005 & 0.30 & 0.01667 \\
\hline ADOBE & 0.20 & 0.95 & 0.2105 \\
\hline ENLUCIDO DE YESO & 0.005 & 0.30 & 0.01667 \\
\hline $\begin{array}{c}\text { Capa de aire superficial } \\
\text { externa }\end{array}$ & -------------- & 0.45384 \\
\hline Total & 0.21 & & 0.45 \\
\hline
\end{tabular}

Nota: Gónzalo, Manual de Arquitectura Bioclimática.

El coeficiente de transmitancia térmica " $\mathrm{K}$ " para la pared es:

$\mathrm{K}=1 / \mathrm{R} \quad \mathrm{K}=1 / 0.45384 \quad\left[\mathrm{~W} / \mathrm{m}^{2} \mathrm{~K}\right]$

$\mathrm{K}=2.2034 \quad\left[\mathrm{~W} / \mathrm{m}^{2} \mathrm{~K}\right]$

\section{El ladrillo cocido como material contemporáneo en la construcción}

El Cantón Chambo en la Provincia de Chimborazo es el corazón de la producción de ladrillo cerámico macizo en el centro del país, la totalidad de piezas monolíticas provienen de esta zona. Su producción artesanal, el bajo costo y la disponibilidad continua hacen de este el material más usado en construcciones contemporáneas.

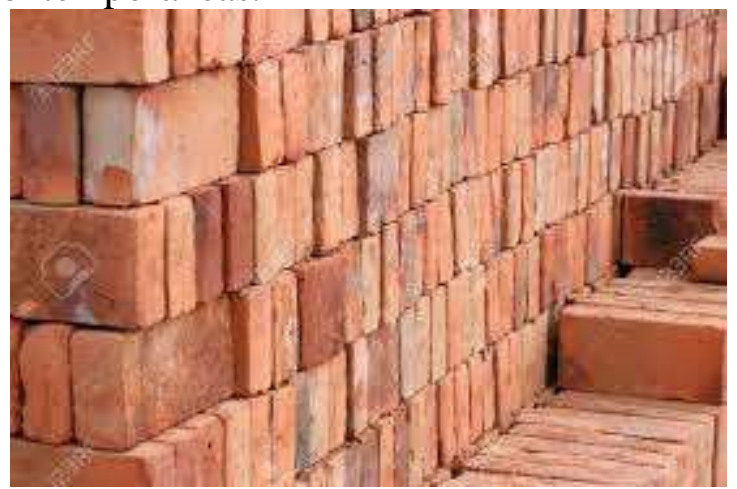

Fig. 4. Ladrillo cerámico macizo, Chambo - Provincia de Chimborazo

Analizando las características del material se confirma que tiene procesos artesanales en todas sus etapas de producción que derivan en una asimetría marcada entre cada una de las piezas monolíticas producidas, deformaciones y diferentes tonalidades dentro de un mismo lote de producción. Estas son algunas de las razones para el deficiente acabado en el plano estético.

La gran cantidad de energía calórica requerida para la producción de este elemento en la etapa de cocción de las unidades, producidas en hornos 
artesanales causa en gran medida afectaciones al ambiente antes, durante y después del proceso de elaboración de cada pieza, convirtiéndolo en un material poco amigable con el ambiente.

Un factor importante en la serranía Ecuatoriana es la variación de temperaturas a lo largo del día, pasando de un leve verano a mitad del día a un invierno inclemente en la madrugada. Este fenómeno poco usual en otras latitudes de la tierra presenta muchas veces en construcciones contemporáneas un problema de confort térmico en estructuras concebidas sin materiales vernáculos. Para determinar su conductividad térmica definimos:

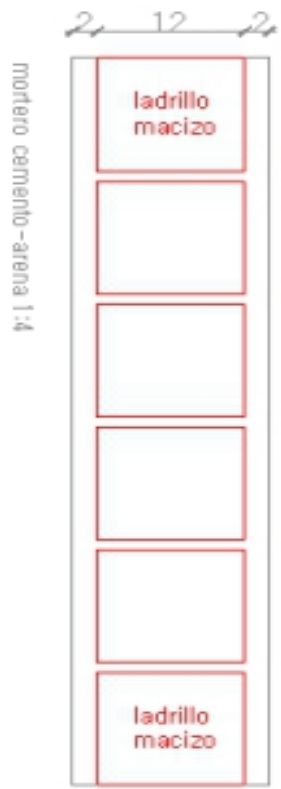

Fig. 5. Detalle constructivo envolvente Paredes e=16cm - mampostería de ladrillo cerámico macizo

\section{Fabrica ladrillo cerámico macizo}

Determinando del coeficiente de transmitancia térmica " $\mathrm{K}$ ":

$\mathrm{K}=1 / \mathrm{R}$

$\mathrm{R}=(1 / \alpha 1+\mathrm{e} 1 / \lambda 1+\mathrm{e} 2 / \lambda 2+\mathrm{e} 3 / \lambda 3+1 / \alpha \mathrm{e})$

Dónde:

$\mathrm{e} 2 / \lambda 2=\mathrm{e} 3 / \lambda 3$ por método constructivo

$\alpha 1=$ Resistencia Superficial Interna

$\alpha e=$ Capa de aire superficial externa

Tabla 5. Valores de conductividad térmica

\begin{tabular}{|l|l|c|}
\hline Capa de elemento constructivo & Espesor & Conductividad térmica
\end{tabular}

(Paredes)

$$
\mathrm{e}(\mathrm{m})
$$
$\lambda(\mathrm{W} / \mathrm{mK})$

FUENTE NBE-CT-79
Resistencia al paso del calor

$\mathrm{R}\left(\mathrm{W} / \mathrm{m}^{2} \mathrm{~K}\right)$ 


\begin{tabular}{|c|c|c|c|}
\hline Resistencia superficial interna & $\begin{array}{l}\text {----------------- } \\
\end{array}$ & ----------------- & 0.17 \\
\hline $\begin{array}{c}\text { Revoque interior. Mortero } \\
\text { Cemento y arena Dosificación } \\
\text { 1:4 (humedad } 5 \mathrm{~g} / 100 \mathrm{~g}) \\
\text { MORTERO DE CEMENTO }\end{array}$ & 0.02 & 1.40 & 0.0142857143 \\
\hline Ladrillo Macizo & 0.12 & 0.87 & 0.1379310345 \\
\hline $\begin{array}{c}\text { Revoque exterior Mortero } \\
\text { Cemento y arena Dosificación } \\
1: 4 \text { (humedad } 5 \mathrm{~g} / 100 \mathrm{~g} \text { ) } \\
\text { MORTERO DE CEMENTO }\end{array}$ & 0.02 & 1.40 & 0.0142857143 \\
\hline $\begin{array}{c}\text { Capa de aire superficial } \\
\text { externa }\end{array}$ & ----------------- & |---------------- & 0.04 \\
\hline Total & 0.16 & & 0.37636 \\
\hline
\end{tabular}

Nota: Gónzalo, Manual de Arquitectura Bioclimática.

El coeficiente de transmitancia térmica " $\mathrm{K}$ " para la pared es:

$\mathrm{K}=1 / \mathrm{R} \quad \mathrm{K}=1 / 0.37636 \quad\left[\mathrm{~W} / \mathrm{m}^{2} \mathrm{~K}\right]$

$\mathrm{K}=2.6570 \quad\left[\mathrm{~W} / \mathrm{m}^{2} \mathrm{~K}\right]$

Después de obtener los valores de $\mathrm{K}$ en los dos muros observamos que, los valores de transmisión térmica del muro de adobe y yeso $\mathrm{K}=2.2034$ [W/ $\mathrm{m}^{2} \mathrm{~K}$ ] son levemente menores a los de la mampostería de ladrillo y enlucido de mortero $\mathrm{K}=2.6570$ [W/ $\mathrm{m}^{2} \mathrm{~K}$ ], por cuanto se concluye que tiene mayor inercia térmica. Una característica definitoria en un ecosistema con un clima variable en la madrugada de la serranía ecuatoriana. La inercia térmica baja de los ladrillos cerámicos ya ha presentado problemas de habitabilidad en los programas de vivienda desarrollados en la serranía ecuatoriana, debido a las bajas temperaturas en la madrugada de paramos ecuatoriales.

Para corroborar los datos obtenidos se eligió una zona definida en la Estructura del Centro Cultural, \{Bodega General $25 \mathrm{~m} 2$ \} usando Ecotect Analisis, modelado específicamente con los dos tipos de envolventes bajo condiciones climáticas correspondientes en la misma latitud y longitud detallados para la zona en Climate Consultant 5.5. 


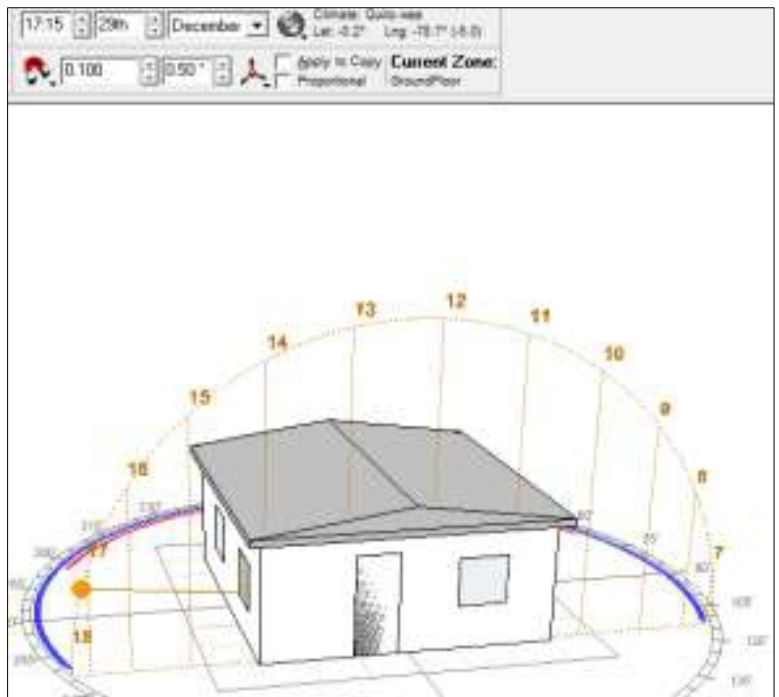

Fig. 6. Modelación de la zona "Bodega general" - Ecotect análisis

En la figura detallada puede verse la incidencia del sol a lo largo del día en la estructura en la latitud y longitud correspondientes a la sierra central ecuatoriana. Su correcta orientación incide en la ganancia total de radiación disponible que en este caso se obtiene al tener el mayor número de ventanales orientados en el eje Este - Oeste.

Para completar la envolvente en techos y pisos se escogieron las mismas condiciones para los dos casos, techos de galvalumen con cámara de aire en estera que evita de mejor manera las perdidas por dicha zona.

La siguiente Fig. representa valores porcentuales de pérdidas y ganancias respecto al total de radiación disponible y el comportamiento global de la estructura cuya envolvente está hecha de ladrillo cocido macizo. La correcta orientación hace que las ganancias por (Sol-Air) sean de $85 \%$ pero, las pérdidas por la envolvente llegan al 39.2\% generadas en gran medida por conducción en los materiales de cerramiento.

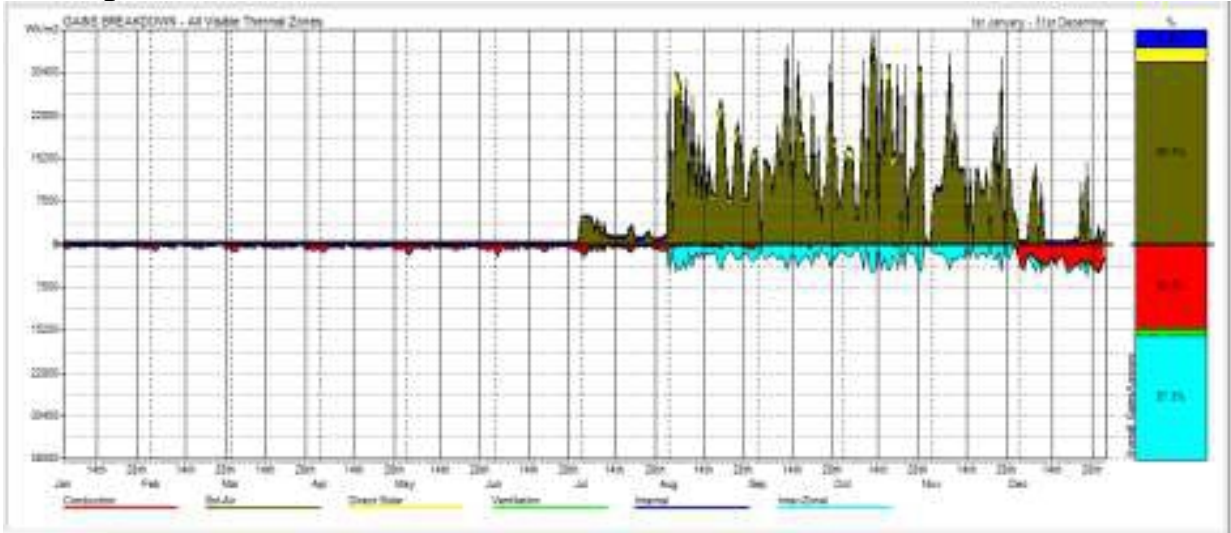

Fig. 7. Valores porcentuales de pérdidas y ganancias 
Tabla 6. Ganancias y pérdidas globales de estructura - Todas las zonas de la estructura Vivienda MIDUVI

\begin{tabular}{|c|c|c|}
\hline Categoría & Pérdidas & Ganancias \\
\hline Fabrica & $39.2 \%$ & $0.0 \%$ \\
\hline Sol-Air & $0.0 \%$ & $85.3 \%$ \\
\hline Solar & $0.0 \%$ & $6.2 \%$ \\
\hline Ventilación & $3.5 \%$ & $0.0 \%$ \\
\hline Internal & $0.0 \%$ & $8.2 \%$ \\
\hline Inter-Zonal & $57.3 \%$ & $0.2 \%$ \\
\hline
\end{tabular}

Con el uso de bloques monolíticos de abobe $40 \mathrm{~cm}$ en la en el cerramiento exterior de la bodega Las pérdidas por la envolvente de la estructura se reducen en $9 \%$, disminuyen del $39.2 \%$ al $31.2 \%$, mientras que la ganancia solar se incrementa en $7 \%$ con respecto a la vivienda no intervenida llegando a valores de $91.4 \%$.

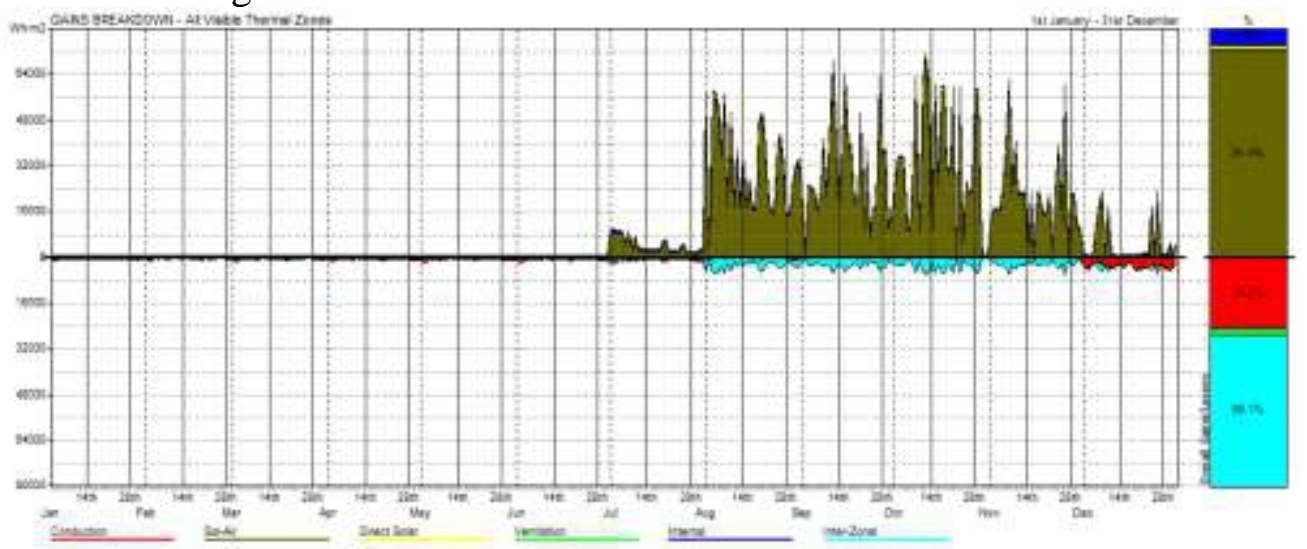

Fig. 8. Comportamiento térmico después de intervención pasiva - todas las zonas de la estructura

\section{Otras adecuaciones para el uso de recursos en el Centro Cultural}

Referente al uso de recursos naturales que se pueden utilizar en la construcción y otras adecuaciones del Centro Cultural en la ESPOCH son varias, como por ejemplo la aplicación de techos verdes, el uso de energía solar, el uso del agua de lluvia, la implementación de una huerta orgánica para la posterior adquisición de los productos sembrados. Todas estas adecuaciones tienen por objetivo optimizar el área de la construcción y convertir el Centro Cultural en una edificación sostenible, siendo un ejemplo para otros inmuebles y que demuestre su eficacia en el diario vivir.

\section{Techos verdes}

Los techos verdes además de influir en el mejoramiento del clima de la ciudad, también optimizan la aislación térmica, el almacenamiento de calor del edificio, y su aislación acústica. Además son considerados, a largo 
plazo, más económicos que las cubiertas convencionales. Los techos enjardinados conducen, en esencia, a una construcción ecológica y económica. Algunos beneficios de los techos verdes son:

- $\quad$ Disminuyen las superficies pavimentadas.

- $\quad$ Producen oxígeno y absorben C02,

- Filtran las partículas de polvo y suciedad del aire y absorben las partículas nocivas

- Evitan el recalentamiento de los techos y con ello disminuyen los remolinos de polvo

- $\quad$ Reducen las variaciones de temperatura del ciclo día - noche

- $\quad$ Disminuyen las variaciones de humedad en el aire.

- Tienen una larga vida útil si es correcta su ejecución,

- $\quad$ Surten efecto como aislamiento térmico,

- $\quad$ Protegen de los intensos rayos solares del verano a las habitaciones ubicadas bajo el techo

- $\quad$ Reducen el pasaje de sonido del exterior

- Valen como incombustibles y

- $\quad$ Absorben la lluvia, por lo que alivian el sistema de alcantarillado.

Por último, algo no menos relevante: las hierbas silvestres en el techo verde generan aromas agradables, dan alojamiento a insectos y escarabajos, son estéticos e influyen positivamente en el buen estado de ánimo y en la distensión de las personas (Minke G, 1995, pág. 9, 10).

\section{Usos de energía solar}

En este caso para el usos de la energía solar, se considera adquirir paneles solares y colocarlos estratégicamente en la construcción, un apartado de la tesis referente al Diseño del Centro Cultural y de la construcción en sí, habla sobre la disponibilidad de adquisición y se presupuesta cierto valor para comprar los paneles dentro del estudio financiero. En todo caso queda planificar el uso que se le daría a la energía proveniente de los paneles dentro de la edificación, si bien es cierto los usos más comunes es para la iluminación y también para colocar un espacio de cargas de celulares con energía limpia.

\section{Usos del agua de lluvia}

En cuanto a la utilización del agua de lluvia se refiere, sus usos son extensos, si bien es fácil su recolección dependiendo del volumen de litros que se necesite y el uso que se le piense dar, disponer de un sistema de tuberías y conexiones para el trasporte del agua seria el objeto de estudios y planificación para construir. Además el volumen para la recolección dependería de igual manera de la disponibilidad del espacio y del material 
que se emplee, considerando las condiciones atmosféricas del área en donde será construido el Centro Cultural. Para este espacio, se considera que la mejor utilización del agua de lluvia podría ser para: expender a los baños públicos y privados, para el riego de la huerta orgánica y como parte del reservorio de agua en épocas de sequía.

\section{Huerta orgánica}

En la Escuela de Ingeniería se han presentado en varias ocasiones proyectos para la construcción de una huerta orgánica, sin embargo la falta de recursos económicos y de personal capacitado, han sido un impedimento para que este proyecto continúe en cada periodo académico. En este preámbulo se han expuesto los impedimentos para la construcción de una huerta orgánica pero, también se debe considerar que estos aspectos pueden mejorar, los recursos se pueden adquirir mediante la presentación de un plan operativo anual POA a la planificación de la Universidad y contar con el apoyo técnico de capacitaciones y personal para el manejo de la huerta. De igual manera los alumnos son quienes se han comprometido en previas ocasiones para cuidar de la huerta, en este caso se necesitará una planificación semestral por cursos para mantener en buen estado la huerta orgánica y atendida constantemente.

Para la construcción es necesario disponer de un espacio de terreno fértil y en el caso de no ser así, prepararlo previamente para sembrar los productos, se considerarán productos de la zona y de consumo para la cafetería del Centro Cultural. A la vez se proyectará mantener estos productos con el compostaje producido por los mismos cuidadores.

El compostaje es un componente especial formado por materiales frescos de origen agrícola micro y macro organismos que ayudan a la fertilización del suelo y colaboran con distintos tipos de nutrientes ya sean minerales y demás que ayudan a que el suelo se mantenga fertilizado, previene la erosión y colabora a que las plantas crezcan de manera saludable (Román, Martínez y Pandoja, 2013, pág. 21).

\section{Conclusiones}

Los valores de transmitancia térmica del muro de adobe enfoscado con yeso $\mathrm{K}=2.2034\left[\mathrm{~W} / \mathrm{m}^{2} \mathrm{~K}\right]$ son levemente menores a los de la mampostería de ladrillo y enlucido de mortero $\mathrm{K}=2.6570\left[\mathrm{~W} / \mathrm{m}^{2} \mathrm{~K}\right]$ por cuanto tiene mayor inercia térmica. Una característica definitoria en un ecosistema con un clima variable en la madrugada de la serranía ecuatoriana con su capacidad de retener el calor en el día y poder liberarlo en la noche.

La correcta orientación, con un mayor número de ventanales dirigidos sobre el eje Este- Oeste para esta latitud, incrementa la ganancia solar en un $7 \%$ en comparación a la estructura no orientada. 
La estructura cuyo cerramiento está hecho con piezas de adobe monolítico reduce sus pérdidas por la envolvente en un $9 \%$ en comparación con el cerramiento de ladrillo cocido macizo.

\section{References:}

1. Acosta D. (2003). Arquitectura y construcciones sostenibles; conceptos, problemas y estrategias.

2. Acosta D. (2009). Arquitectura y construcciones sostenibles; conceptos, problemas y estrategias. Disponible en http://www.redalyc.org/pdf/3416/341630313002.pdf. Consultado el 27 de mayo del 2017

3. Bedoya C. (2011). Construcción sostenible, para volver al camino. Biblioteca jurídica Dike-UNECO. Colombia.

4. Comisión de carrera de la EIE. (2012). Objetivo, misión y visión de la Escuela de Ingeniería en Ecoturismo. Disponible en https://www.espoch.edu.ec/images/facultades/recursos_naturales/arch ivos/inf curricular EIE.pdf. Consultado el 20 de noviembre del 2016.

5. Construcciones a base de tierra. Revista Informes de la Construcción. Instituto Eduardo Torroja. Vol. 36 No 365. Madrid, 1984. pág. 47-51.

6. Del Río M., Sainz A. (2011). La evolución de los sistemas constructivos en tierra. Construcción con Tierra, Tecnologías y arquitectura. Universidad de Valladoli. pág. 56-70.

7. Florensa R., Coch H. (1995). Arquitectura y energía natural. Edición de la Universidad Politécnica de Cataluya. España.

8. Lodeiro T. (2008). Consumir menos, vivir mejor. Ideas prácticas para un consumo más consiente. Editorial Txalaparta, España.

9. Morales R., Cabrejos R., Rengifo L., Candiotti C. (1993). Manual para la construcción de viviendas en adobe. Universidad Nacional de Ingeniería. Perú.

10. Minke G. (1995). Techos verdes; Planificación, ejecución, consejos prácticos. Editorial Fin de Siglo. Alemania. pág. 9-11.

11. Minke G. (2001). Manual de construcciones para viviendas antisísmicas de tierra. Universidad de Kassel. Alemania.

12. Oshiro F. (1990). Construcción de vivienda económica en adobe estabilizado. PREDES. Perú. pág. 12-16

13. Román P., Martínez M., Pandoja A. (2013). Manual de Compostaje del Agricultor; Experiencias en América Latina. FAO. Santiago de Chile. pág. 21 
14. Tresserras J. (2012). Labels de los Productos - Mercado del Turismo Cultural. Módulo de turismo cultural, educación y ciudadanía. Universidad de Barcelona. España

15. UNESCO. (2016). Informe mundial sobre la cultura para el desarrollo urbano sostenible. Hábitat III, UNESCO. Francia.

16. UNESCO. (2005). El concepto de sostenibilidad. Disponible en: http://unesdoc.unesco.org/images/0016/001621/162177s.pdf. Consultado el 15 de diciembre del 2016. 